\title{
Experimental and theoretical study of bumped characteristics obtained with cylindrical Langmuir probe in magnetized Helium plasma
}

\author{
J. Ledig, ${ }^{1}$ E. Faudot, ${ }^{1}$ J. Moritz, ${ }^{1}$ S. Heuraux,${ }^{1}$ N. Lemoine, ${ }^{1}$ and M. Usoltceva ${ }^{1,2}$ \\ ${ }^{1)}$ Université de Lorraine - Institut Jean Lamour, Campus Artem 2 allée André Guinier - BP 50840, \\ 54011 NANCY Cedex, France \\ 2) Max Planck Institut für Plasmaphysik - Boltzmannstr. 2, 85 748, Garching, \\ Germany
}

Cylindrical Langmuir probe measurements in a Helium plasma were performed and analysed in the presence of a magnetic field. The plasma is generated in the ALINE device, a cylindrical vessel 1 $\mathrm{m}$ long and $30 \mathrm{~cm}$ in diameter using a direct coupled $\mathrm{RF}$ antenna $\left(\nu_{\mathrm{RF}}=25 \mathrm{MHz}\right)$. The density and temperature are of the order of $10^{16} \mathrm{~m}^{-3}$ and $1.5 \mathrm{eV}$, respectively, for $1.2 \mathrm{~Pa}$ Helium pressure and $200 \mathrm{~W}$ RF power. The axial magnetic field can be set from 0 up to $0.1 \mathrm{~T}$, and the plasma diagnostic is a RF compensated Langmuir probe, which can be tilted with respect to the magnetic field lines. In the presence of a magnetic field, $I(V)$ characteristics look like asymmetrical double probe ones (tanh-shape), which is due to the trapping of charged particles inside a flux tube connected to the probe on one side and to the wall on the other side. At low tilting angle, high magnetic field amplitude, power magnitude and low He pressure, which are the parameters scanned in our study, a bump can appear on the $I(V)$ in the plasma potential range. We then compare different models for deducing plasma parameters from such unusual bumped curves. Finally, using a fluid model, the bump rising on the characteristics can be explained, assuming a density depletion in the flux tube, and emphasizing the role of the perpendicular transport of ions.

\section{I. INTRODUCTION}

10 Cylindrical Langmuir probes are one of the sim11 plest device to investigate plasma properties as they 12 consist of a small metallic wire of length $L_{p}$ and ${ }_{13}$ radius $r_{p}$, usually made of tungsten, immersed into ${ }_{14}$ the plasma, and submitted to a ramp of voltage. The 15 collected current by the tip vs. the applied voltage ${ }_{16}$ yields an $I(V)$ probe characteristics, from which ${ }_{17}$ electron density $n_{e}$, ion density $n_{i}$ and temperature ${ }_{18} T_{e}$ can be derived.

${ }_{19}$ An $I(V)$ curve can be divided in three parts : 20 the "ion saturation current" part, the "electron sat${ }_{21}$ uration current" and the exponential part ${ }^{1,2}$. For ${ }_{22}$ strongly negative potentials $V$ applied to the probe ${ }_{23}$ (with respect to plasma potential $\phi_{p}$ ) electrons are 24 repelled and ions accelerated towards the probe, the ${ }_{25}$ collected current being the ion saturation current $I_{i}$. ${ }_{26}$ In the opposite case, $V \gg \phi_{p}$, only electrons are ${ }_{27}$ collected and the measured current at the probe is ${ }_{28}$ the electron saturation one $I_{e}$. These regions are so ${ }_{29}$ called "saturation current" because their mean ve30 locities saturate at $\langle v\rangle_{\max }$, deduced from their veloc31 ity distribution. Actually even in the saturation part, ${ }_{32} I$ keeps on increasing with $V$, because the sheath 33 surrounding the probe is growing with the applied ${ }_{34}$ potential. Thus, the collecting surface for the accel35 erated species in the sheath is not the probe surface, 36 but the sheath one. Within the transition region, ${ }_{37}$ electrons are repelled according to the Boltzmann ${ }_{38}$ factor and $n_{e} e^{-E / k_{B} T_{e}}$, with $E=-e\left(V-\phi_{p}\right)$. An39 other important point of the $I(V)$ characteristic is 40 the floating potential, $\phi_{\mathrm{fl}}$, defined as the probe po-
${ }_{41}$ tential for which the same amount of ion and elec42 tron are collected, i.e. for $I=0$. Note that the con43 vention is to count ion current as negative, and elec44 tron current as positive on $I(V)$ plots.

Determining the plasma parameters on differ${ }_{46}$ ent regions listed above requires to use the most 47 appropriate theory for each species. Mott-Smith ${ }_{48}$ and Langmuir ${ }^{3}$ proposed the first model to extract 49 temperature and density from characteristics using ${ }_{50}$ the OML theory (Orbital Motion Limited). This ${ }_{51}$ theory exploits mainly the ion part of the charac52 teristics and was developed with the assumption 53 of large sheaths $\left(r_{p} / \lambda_{\mathrm{D}} \ll 1\right.$, for $\lambda_{\mathrm{D}}$ the Debye ${ }_{54}$ length of the repelled species), large ion mean-free${ }_{55}$ path $\left(\lambda_{i, \mathrm{mfp}} / L_{p} \ll 1\right)$ and cold ions $\left(T_{i} / T_{e} \rightarrow 0\right)$. ${ }_{56}$ Allen and Bernstein ${ }^{4-6}$ improved this theory, solv${ }_{57}$ ing the Poisson equation within the sheath, which ${ }_{58}$ was omitted in the OML theory. But it can lead to 59 an overestimation of the ion density by a factor of 60 ten ${ }^{7}$. Laframboise extended the model assuming a ${ }_{61}$ velocity distribution function for ions ${ }^{8}$, but this so62 phisticated approach does not improve the fits of the ${ }_{63}$ experimental ion current with respect to the ABR ${ }_{64}$ (Allen Boyd Reynolds) model ${ }^{5}$.

The presence of a magnetic field changes ${ }_{66}$ strongly the way particles are collected on the 67 probe: the motion of charged particles can be di${ }_{68}$ vided into a longitudinal $(\| \mathbf{B})$ and a perpendic69 ular $(\perp \mathbf{B})$ components, with their own tempera70 ture. In such magnetized conditions, OML the${ }_{71}$ ory still holds ${ }^{9-11}$ for ions, but the electron part is 72 hardly interpretable ${ }^{12}$ due to the distortion of the ${ }_{73} I(V)$ curve, leading to an uncertainty on the deter- 


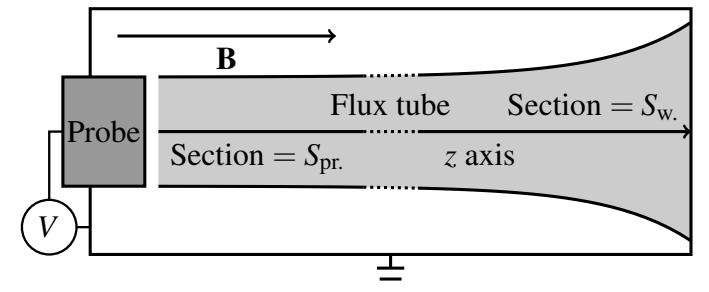

FIG. 1. Illustration of the double probe model : the widening of the flux tube is here to model the fact that at the end of the vessel magnetic field lines drive away each other.

74 mination of $\phi_{p}$ and, thus, to a wrong $T_{e}$ and $n_{e}$. Sev${ }_{75}$ eral authors ${ }^{11,13}$ emphasized the distortion of the ${ }_{76}$ characteristics in the presence of $\mathbf{B}$, showing that ${ }_{77} I_{e}$ is much lower compared to the unmagnetized ${ }_{78}$ case, because electrons are stuck along magnetic 79 field lines, with a low level of perpendicular trans${ }_{80}$ port due to collisions ${ }^{14}$ and drain diffusion ${ }^{11}$ for in81 stance.

${ }_{82}$ In several papers it was also reported that, in 83 some cases, a bump on the characteristics can ex${ }_{84}$ ist between the exponential part and the electron ${ }_{85}$ saturation part ${ }^{15-18}$. It was assumed that the bump 86 was caused by a density depletion of the flux tube ${ }_{87}$ during the probing. Dote developed an OML-like ${ }_{88}$ model to explain the presence of the bump ${ }^{19,20}$ and 89 suggested the plasma potential to be the bump posi90 tion; his model however does not match quite well ${ }_{91}$ with experimental results.

${ }_{92}$ The shape of a $I(V)$ characteristic in a mag${ }_{93}$ netized plasma can be approached, excluding the 94 eventual bump, by a double probe model: the per95 fectly confined flux tube (which is $\| \mathbf{B}$ ) is connected ${ }_{96}$ to one hand to the probe, and on the other hand, to ${ }_{97}$ the wall of the reactor as shown in Fig. 1.

${ }_{98}$ The collected electron current is mainly paral99 lel to $\mathbf{B}$ while the ion current is perpendicular to ${ }_{100}$ B so that the lateral surface of the flux tube plays 101 the role of the wall in a classic unmagnetized dis102 charge. The magnetized double probe model can 103 then be seen as a classic asymmetric double pobe 104 model without magnetic field ${ }^{21}$. The effect of these 105 ionic perpendicular currents both in $\mathrm{DC}^{22,23}$ and in ${ }_{106} \mathrm{RF}^{24,25}$ have already been studied for planar probes. ${ }_{107}$ It was shown that the shape of the $I(V)$ curve was 108 changed by feeding or pumping the flux tube and 109 that I-Vs are really sensitive to the $r_{p} / r_{L}$ ratio.

110 Here simple asymmetric model is $1 \mathrm{D}$ in the $z$ di111 rection (see sketch fig.1), the probe is located at ${ }_{112} z_{\text {pr. }}=0$ and the wall at $z_{\mathrm{w} .}=L_{t}$, the length of the ${ }_{113}$ flux tube is then $L_{t}$ and $\mathbf{B}=B \mathbf{e}_{z}$. The probe po${ }_{114}$ tential is at $V$, the space potential is $\phi_{t}$ and the wall 115 is grounded. The section of the tube on the wall ${ }_{116}$ side is $S_{\mathrm{w}}$, and the section at the probe is $S_{\mathrm{pr}}$ with
${ }_{117} S_{\text {pr. }} \leq S_{\mathrm{w} .}$. We assume constant density in the tube, ${ }_{118} n_{t}$, and no loss in the perpendicular direction. Thus, 119 the stationary $\left(\partial_{t} n=0\right)$ continuity equation writes :

$$
\boldsymbol{\nabla} \cdot \mathbf{J}_{\text {Tot. }}=0 \quad \text { where } \mathbf{J}_{\text {Tot. }}=\mathbf{J}_{e}+\mathbf{J}_{i}
$$

120 For homogeneous current density across both ends, 121 using Gauss's theorem by integrating eq.(1) over 122 the whole flux tube gives :

$$
J_{\text {Tot. }}(z=0) \times S_{\text {pr. }}+J_{\text {Tot. }}\left(z=L_{t}\right) \times S_{\text {w. }}=0
$$

Ion current density is the Bohm flux,

$$
J_{i}=0.61 \times e n_{t} c_{s} \quad \text { where } c_{s}=\sqrt{\frac{k_{B} T_{e}}{m_{i}}},
$$

and for electron it is given the Boltzmann equilibrium with the local potential,

$$
\begin{aligned}
J_{e}=-\frac{1}{4} e n_{t}\left\langle v_{e}\right\rangle \times \exp \left[e \frac{\phi(z)-\phi_{t}}{k_{B} T_{e}}\right] \\
\text { where }\left\langle v_{e}\right\rangle=\sqrt{\frac{8 k_{B} T_{e}}{\pi m_{e}}} .
\end{aligned}
$$

23 Introducing the electron saturation current as ${ }_{24} J_{e, \text { sat. }}=e n_{t}\left\langle v_{e}\right\rangle / 4$ and the floating potential, $\phi_{\mathrm{fl} .}=$ ${ }_{125} k_{B} T_{e} \ln \left(J_{i} / J_{e, \text { sat. }}\right) / e$, eq. (2) becomes :

$$
\phi_{t}=\frac{k_{B} T_{e}}{e} \ln \left[\frac{\Sigma+\exp \left(e V / k_{B} T_{e}\right)}{\Sigma+1}\right]-\phi_{\mathrm{fl} .},
$$

126 where $\Sigma=S_{\mathrm{w} .} / S_{\mathrm{pr} .}$. Finally, the collected current on 127 the probe is

$$
J_{\text {pr. }}(V)=J_{e, \text { sat. }} \exp \left[e \frac{V-\phi_{t}(V)}{k_{B} T_{e}}\right]-J_{i} .
$$

${ }_{128}$ Thus, using eq.(3) in eq.(4) one will get :

$$
J_{\text {pr. }}(V)=J_{i} \times \frac{\exp \left(e V / k_{B} T_{e}\right)-1}{1+\frac{1}{\Sigma} \exp \left(e V / k_{B} T_{e}\right)}
$$

129 The asymmetric double probe $I(V)$ characteristics 130 from eq.(5) is plotted in fig. 2.

In this paper, we investigate the general be132 haviour of "bumped characteristics" with respect 133 to several parameters, such as the amplitude of the ${ }_{134}$ magnetic field, the gas pressure or the RF power 135 input. We also propose a new explanation of 136 the bump, with the aim of a better understanding ${ }_{137}$ of Langmuir probe measurements in magnetized 138 plasma. In the first part sect.II, the experimental 139 set-up and the plasma parameters (mean free paths, ${ }_{140}$ Larmor radii, etc.) are detailed. Then the main ex${ }_{141}$ perimental results are shown in section III, where 142 the behaviour of the bumps was studied with respect 143 to the amplitude of the magnetic field in sect.III A, 144 the angle $\vartheta$ between the probe and $\mathbf{B}$ in sect.III B, ${ }_{145}$ the RF-power input in sect.III C, the probe position 


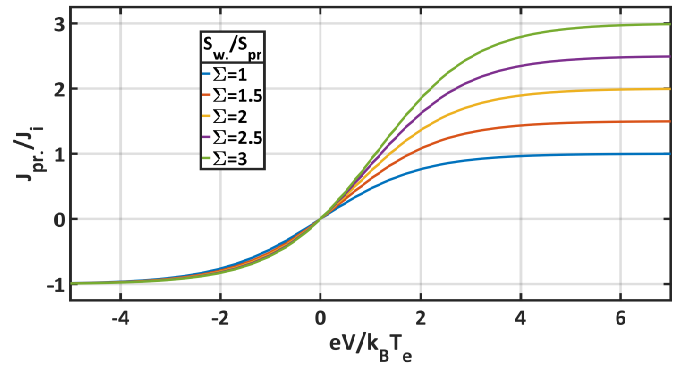

FIG. 2. Theoritical and normalized double probe characteristics for several values of $\Sigma$. For $\Sigma \neq 1$ the characteristics are called "assymetric", and for $\Sigma \rightarrow \infty$ they are very similar to classical Langmuir characteristics $\left(S_{\mathrm{w}}\right.$ is the surface of the whole vessel in that case).

146 with respect to the RF-antenna in sect.III D and fi${ }_{147}$ nally the He pressure in sect.IIIE. In the follow148 ing, sect.III, a method is proposed to determine the 149 plasma temperature and density with conventional 150 methods (when no magnetic field is present). Fi151 nally, the origin of the bump characteristics is ex152 plained thanks to a fluid model in the last section.

\section{II. EXPERIMENTAL SETUP}

${ }_{154}$ Experiments were performed in the ALINE ${ }^{26,27}$ 155 (A LINEar plasma device) reactor (see figure 3 and 156 4). The cylindrical chamber is $1 \mathrm{~m}$ long and $30 \mathrm{~cm}$ ${ }_{157}$ diameter. The typical discharges presented here are ${ }_{158}$ generated by a RF-antenna at $v_{\mathrm{RF}}=25 \mathrm{MHz}$ (but 159 the amplifier frequency can be tuned from $10 \mathrm{kHz}$ 160 to $250 \mathrm{MHz}$ ), and the RF-power is in the range 20 $161-200 \mathrm{~W}$ (though 0 to $600 \mathrm{~W}$ is achievable). The 162 amplifier is directly connected to the antenna (direct 163 coupling, so the average potential on the antenna is ${ }_{164}(\mathrm{~V})$. The cathode is at the center of the vessel has 165 a radius of $4 \mathrm{~cm}$ and is $1 \mathrm{~cm}$ thick.

166 Six circular coils generate an axial magnetic field ${ }_{167}$ from 0 to about $100 \mathrm{mT}$ (the current in the coils is ${ }_{168}$ in the range 0-200 A). Helium gas was used for ${ }_{169}$ all discharges with a pressure in the range between 1701.2 and $40 \mathrm{~Pa}$ for this study, which allows the study 171 from collisionless to collisional regimes.

${ }_{172}$ The cylindrical Langmuir probe Tungsten tip 173 used in measurements has a length $L_{p}$ of $1 \mathrm{~cm}$ and a ${ }_{174}$ radius $r_{p}$ of 75 microns. To enable measurements in 175 a RF plasma, the probe is RF-compensated ${ }^{7,28}$. For 176 each $I(V)$ characteristics, a voltage ramp from -70 177 to $70 \mathrm{~V}$ is swept 20 times at a frequency of the or178 der of $65 \mathrm{kHz}$. Hence, the measurement frequency 179 is much slower than RF-oscillations and all plasma 180 frequencies $\left(\omega_{c}\right.$ and $\left.\omega_{p}\right)$, and thus, can be seen as 181 "stationary" with respect to the plasma dynamics.

${ }_{182}$ The position of the probe tip is given with re183 spect to the middle of the antenna $(y=0$ and $z=0)$.

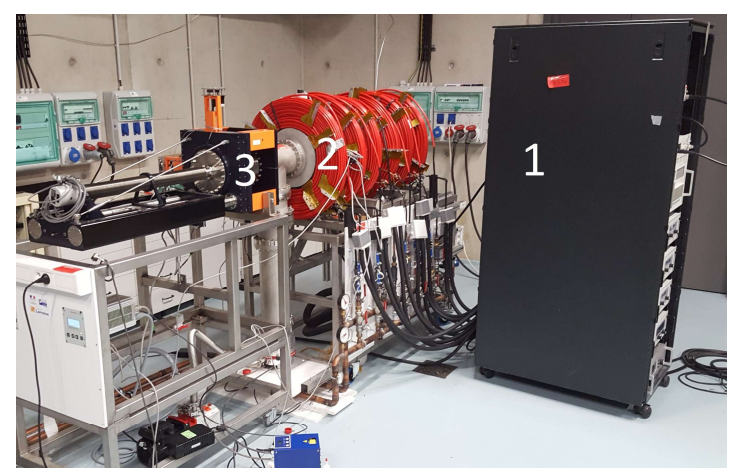

FIG. 3. Photograph of the ALINE plasma device. The cylindrical vessel (2) is $1 \mathrm{~m}$ long and $30 \mathrm{~cm}$ diameter. Six coils (in red) are placed equidistantly along the axis, around the chamber to generate a quasi-homogeneous and uniaxial magnetic field along the axis of the cylinder. The power supplies for the coils and the RF antenna are placed in the rack (1). The antenna is in the middle of the vessel. The arm (3) holding the Hidden Langmuir probe along the vessel's axis was developed by Cryoscan and is able to perform 3D translations (along the axis, up/down and left/right). Note that the arm is always parallel to the axis of the cylinder.

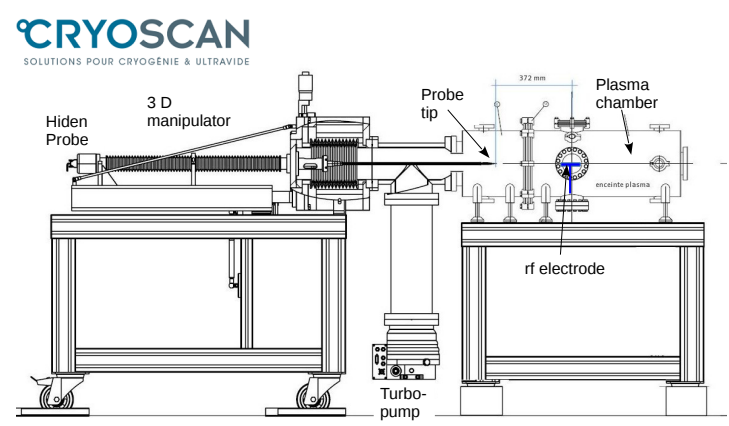

FIG. 4. Schematic representation of the plasma device designed by Cryoscan. The gas inlet is on the top-right end of the device (on the opposite of the pump).

${ }_{184}$ All measurements were performed at $z=-60 \mathrm{~mm}$ 185 along the axis of the cylindrical chamber and $y=40$ ${ }_{186} \mathrm{~mm}$ above the antenna. The arm holding the probe ${ }_{187}$ is parallel to the axis of the cylindrical vessel, and 88 only the tip is tilted $\vartheta$ with respect to the magnetic gield lines (see fig.5). $\vartheta \in[0,6,12,18,40,94]^{\circ}$ an190 gles were used for the study.

Moreover, the arm (see (3) in figure 3) is able 92 to move the probe tip inside a volume (see the red ${ }_{193}$ dashed box in figure 6) to get three-dimensional 194 measurements of plasma parameters. Solving Biot${ }_{195}$ Savart law in the whole vessel gives the magnetic 196 field topology. Figure 6 shows the result of the 197 computation. Let $\langle B\rangle_{\text {meas. }}$ be the averaged modulus 198 inside the workable volume : in this paper we as199 sume uniaxial (along $z$, the axis of the reactor) and 


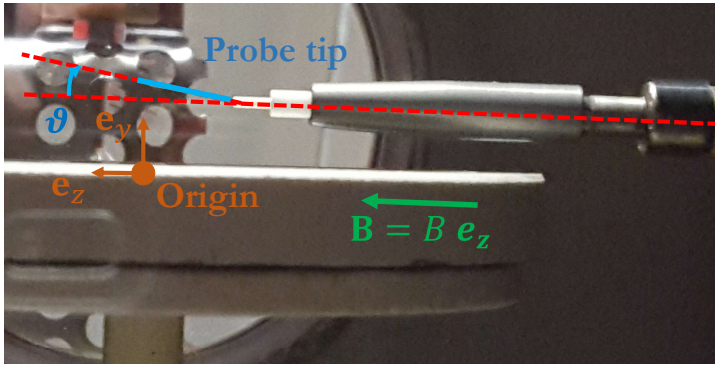

FIG. 5. Tilted cylindrical Langmuir probe with an angle $\vartheta=12^{\circ}$ with respect to $\mathbf{B}$ (which is assumed homogeneous and constant in the whole probed volume, $\mathbf{B}=\mathbf{C t e})$. The position of the probe is $z=0$ and $y=5 \mathrm{~mm}$ on this photograph. The value of the angle with respect to the antenna was measured thanks to the open source GeoGebra software.

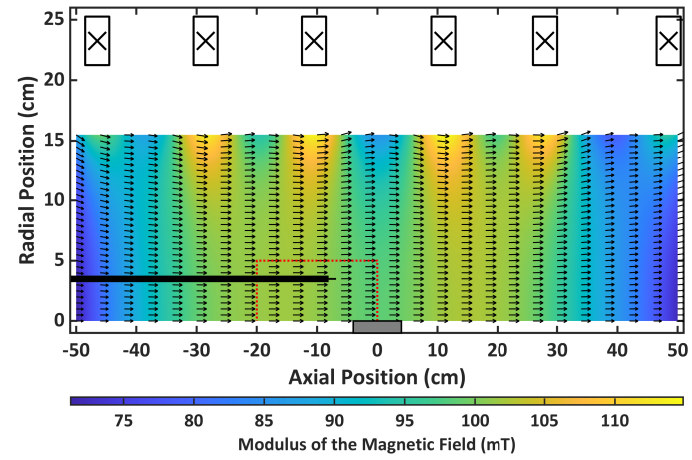

FIG. 6. Magnetic topology in the ALINE plasma device. The gray rectangle at the bottom represents the RF cathode, the long black rectangle and the narrow line at its end at $r=4 \mathrm{~cm}$ represents the probe and its arm at probing position $(x, y, z)=(0,40,-60) \mathrm{mm}$. The red dashed box delimits the workable volume. White arrows represent the local magnetic field vectors.

200 constant magnetic field (the deviation from the av201 eraged value being less than 3\% in the probed vol202 ume). In the following $B=\|\mathbf{B}\|=\langle B\rangle_{\text {meas. }}$, and ${ }_{203} \mathbf{B}=B \mathbf{e}_{z}$.

205 In low pressure conditions, $p=1.2 \mathrm{~Pa}$, the 206 plasma can be considered as collisionless. Indeed 207 after the values listed in table I, electron mean ${ }_{208}$ free path is greater than the probe dimensions ${ }^{6}$, 209 i.e. $\quad \lambda_{e, \mathrm{mfp}} \gg r_{p}$ and $L_{p}$. Ions can be consid210 ered as unmagnetized for the probe since $\rho_{c i} \gg r_{p}$. ${ }_{211}$ Note that an electron needs a parallel velocity over ${ }_{212} L_{p} \omega_{c e} / 2 \pi \approx 2.8 \times 10^{7} \mathrm{~m} / \mathrm{s}$ to overfly the probe 213 without completing a cyclotron period : at this ve214 locity the $f_{e}(\mathbf{v}) \sim 0$, which means that almost all 215 electrons complete an entire turn over the length of ${ }_{216}$ the probe. The electron collection can thus be seen ${ }_{217}$ as the intersection of the "cyclotron disk" $\left(\pi \rho_{c e}^{2}\right)$ ${ }_{218}$ with the probe for parallel inclination in collision219 less regimes.
TABLE I. Plasma parameters for $\|\mathbf{B}\|=100 \mathrm{mT}$ and $p=1.2 \mathrm{~Pa}$. Note that probe dimensions are $r_{p}=75$ $\mu \mathrm{m}$ and $L_{p}=1 \mathrm{~cm}, \rho_{c}$ is the Larmor radius, $\lambda_{\mathrm{mfp}}$ is the mean-free-path for charged particle/neutral collisions, $v_{c}$ the cyclotron frequency $\left(\omega_{c} / 2 \pi\right), v_{p}$ the plasma frequency and $v_{\text {col. }}^{N}$ the charged particle/neutral collision frequency 29,30 .

\begin{tabular}{clcc}
\hline \hline \multicolumn{2}{c}{ Quantity } & Ions He $^{+}$ & Electrons $e^{-}$ \\
\hline$T$ & $(\mathrm{eV})$ & 0.026 & $2-4$ \\
$n$ & $\left(\mathrm{~m}^{-3}\right)$ & $5-50 \times 10^{15}$ & $5-50 \times 10^{15}$ \\
\hline$\rho_{c}$ & $(\mu \mathrm{m})$ & 400 & $37-83$ \\
$\lambda_{\mathrm{mfp}}$ & $(\mathrm{cm})$ & 1.50 & $2-4.5$ \\
\hline$v_{c}$ & $(\mathrm{~Hz})$ & $380 \times 10^{3}$ & $3 \times 10^{9}$ \\
$v_{p}$ & $(\mathrm{~Hz})$ & $7-23 \times 10^{6}$ & $635-2000 \times 10^{6}$ \\
$v_{\text {col. }}^{N}$ & $(\mathrm{~Hz})$ & $88 \times 10^{3}$ & $38-85 \times 10^{6}$ \\
\hline \hline
\end{tabular}

\section{III. EXPERIMENTAL STUDY}

$221 \quad$ Scans over $B, \vartheta, \mathrm{RF}$-power, $y$-position and pres222 sure were performed and main results are presented ${ }_{223}$ here. If not specified the probe tip position is set 224 by default at $y=40 \mathrm{~mm}$ and $z=-60 \mathrm{~mm}$, and the 225 pressure at $1.2 \mathrm{~Pa}$.

\section{${ }_{226}$ A. Influence of the magnetic field}

${ }_{227} I(V)$ Characteristics for all inclinations of the ${ }_{228}$ probe tip have been plotted for several values of ${ }_{229}\|\mathbf{B}\|$ and for a $200 \mathrm{~W}-\mathrm{RF}$ power input in fig.7. With230 out magnetic field (fig.7-(a)), the "classical" $I(V)$ ${ }_{231}$ is recovered, because the plasma is an isotropic ${ }_{232}$ medium and the orientation of the probe unimpor233 tant. The slight differences between all six curves 234 come from small variations on the plasma condi235 tions, due to the fact that the change of inclination 236 requested to open the chamber between each mea237 surement (uncertainties within $5 \%$ due to the gas ${ }_{238}$ pressure gauge, thus the RF coupled power which ${ }_{239}$ is sensitive to the pressure may not be exactly the 240 same).

The shape of the $I(V)$ changes drastically in the 242 presence of a magnetic field as depicted in fig.7243 (b) to (d). The slope of the exponential part and 244 the electron saturation current one as well as the ra245 tio $I_{e} / I_{i}$ are strongly affected by the addition of a 246 magnetic field ${ }^{11}$. Note that the increase of $I_{e}$ with 247 the magnetic field is due to a better coupling of the ${ }_{248} \mathrm{RF}$ power and to better confinements. More gen249 erally, it can be seen that the overall shape of the 250 characteristics are qualitatively close to the dou251 ble probe/tanh-shape ones modelled by eq.(5). For 252 small angles $\left(\vartheta \leq 12^{\circ}\right)$, the characteristics even dis253 play a bump between the exponential and the satu254 ration parts. The bump's overshoot amplitude and 255 the change in the slope between the exponential part 

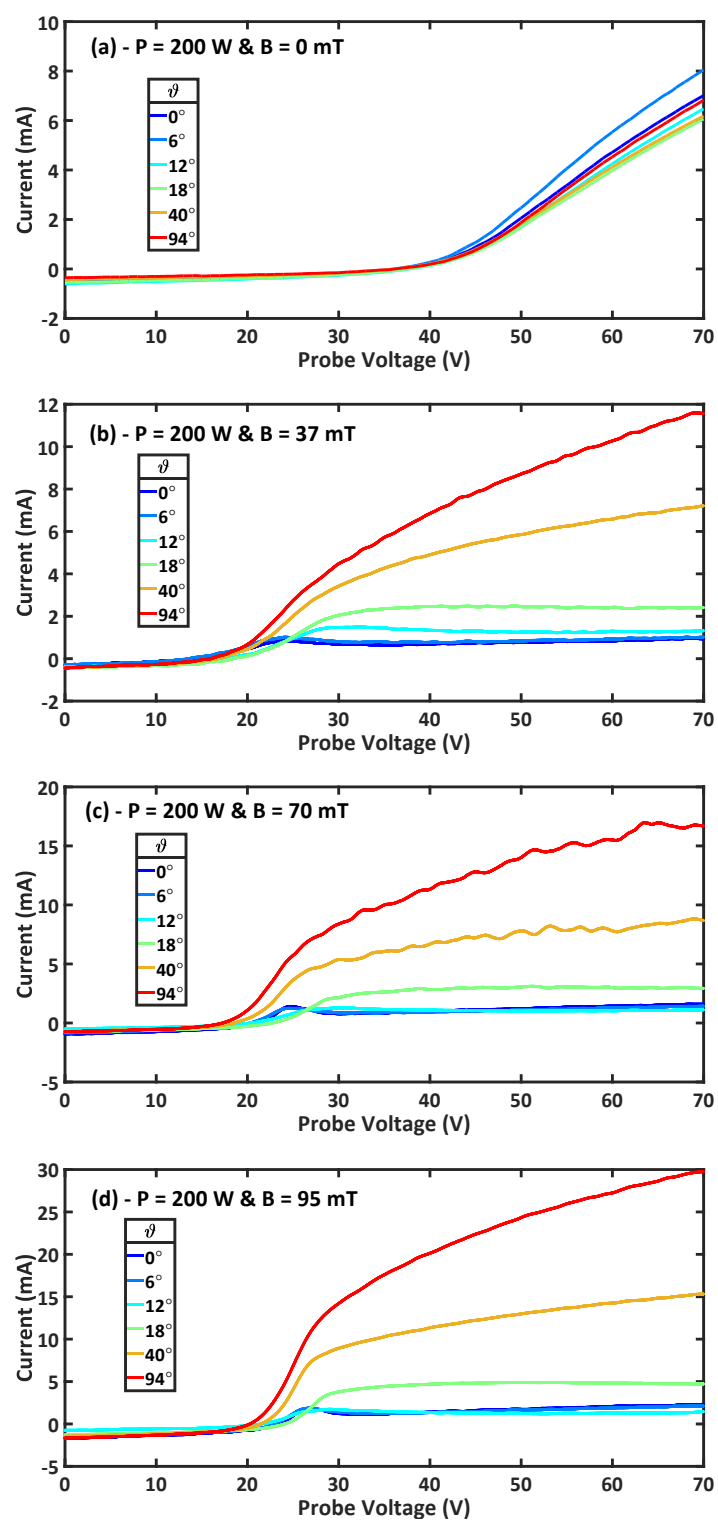

FIG. 7. Evolution of the $I(V)$ characteristics at $200 \mathrm{~W}-\mathrm{RF}$ power, at position $y=40 / z=-60 \mathrm{~mm}$ with increasing ||B $\mid$ from 0 (a) to $95 \mathrm{mT}$ (d) for all six $\vartheta$ inclinations, 1.2 $\mathrm{Pa} \mathrm{He}$. Potential range of the measurements were -70 to $+70 \mathrm{~V}$, but the purpose of the study is not the ion part so only the range $[0,70] \mathrm{V}$ is displayed here. Note that the current range changes for each graphs.

256 and the electron saturation regime is emphasized 257 and steeper with larger $\|\mathbf{B}\|$.

\section{${ }_{258}$ B. Influence of probe inclination}

259 For a probe inclination of $18^{\circ}$, the measured $I(V)$ 260 characteristic looks like the "tanh-shape" as ex261 plained previously. For higher inclination angle, the 262 electron current does not saturate (due to sheath ex-
${ }_{263}$ pansion) and for lower inclination angle, there is a 264 bump. The only difference between all these dif265 ferent cases is the width of the flux tube that scales ${ }_{266}$ as $\sim L_{p} \sin \vartheta$. The probe area facing magnetic field 267 lines (see fig.5) is written as follows :

$$
S_{\text {face }}=\pi r_{p}^{2} \cos \vartheta+\pi L_{p} r_{p} \sin \vartheta
$$

${ }_{268}$ which can be scaled as $S_{\text {face }} \sim \sin \vartheta$ because $L_{p} \gg$ ${ }_{269} r_{p}$.

${ }_{270}$ For $\vartheta=0^{\circ}$ at $100 \mathrm{mT}, r_{p} \approx 2 \rho_{c e}$, therefore, the 271 probe surface facing the magnetic flux tube is com272 parable to the "cyclotron area" $\left(S_{c e}=\pi \rho_{c e}^{2}\right)$ : in 273 this case of grazing incidence, a bump arises on 274 the measured characteristics. By increasing the an275 gle, the facing surface increases (whereas the cy276 clotron area remains constant) and the amplitude of 277 the bump decreases, and even disappears for larger 278 angles. One can suggests that the flux tube nar279 rowness comparable to the cyclotron area could ex280 plain the bump. However, it remains even if $S_{\text {face }} \gg$ ${ }_{281} S_{c e}$ (when $\vartheta>5^{\circ}$ ), therefore another mechanism ${ }_{282}$ should be invoked in order to explain the presence 283 of the bump.

${ }_{284}$ We performed a series of experiments with a 285 power input in the range $20-200 \mathrm{~W}$ in order to 286 quantify the evolution of the characteristics with re287 spect to $\vartheta$. Fig.8 shows the evolution of the current 288 at $70 \mathrm{~V}, I\left(V=V_{\max }=70 \mathrm{~V}\right)$ or the "end-current", 289 against $\sin \vartheta$. This end value is used, because the 290 plasma potential is actually unknown, so the com291 parison of the current at plasma potential is not pos292 sible for now.

Without magnetic field (fig.8-(a)), the end294 current is constant for any inclination as explained 295 previously. Moreover by increasing the RF-power, 296 the overall collected end-current also increases, be${ }_{297}$ cause the power also increases the plasma density ${ }_{298}\left(I \propto n_{e}\right)$ as expected.

${ }_{299}$ In the presence of a magnetic field of $95 \mathrm{mT}$ 300 (fig.8-(b)) two regimes are evidenced : the region 301 where there is a bump $\left(\vartheta \leq 12^{\circ} \Leftrightarrow \sin \vartheta \leq 0.21\right.$ ) 302 and the region with an asymmetric double probe 303 behaviour (above $12^{\circ}$ ). In the former region, the 304 end current is proportional to $\sin \vartheta$, as the width 305 of the magnetic flux tube : the sine dependence of 306 the current collection is verified. But in the "bump 307 region", the collected end-current remains approxi308 matively constant with $\vartheta$ for any RF-power. Since 309 the collected current is proportional to the product 310 of the density with the collecting surface, $n_{e} S_{\text {coll. }}$. 311 (assuming $\left\langle v_{e}\right\rangle \sim\left\langle v_{e, \|}\right\rangle \approx$ Cte.), the increase of the 312 angle also increases $S_{\text {coll. }}$, so to keep constant col${ }_{313}$ lected current, the electron density in the flux tube 314 should decrease. This is in a good agreement with 315 figure 9-(b): in the presence of a magnetic field, and 316 if there is a bump on the characteristic, the density 317 is lower than in the absence of a bump (going from 

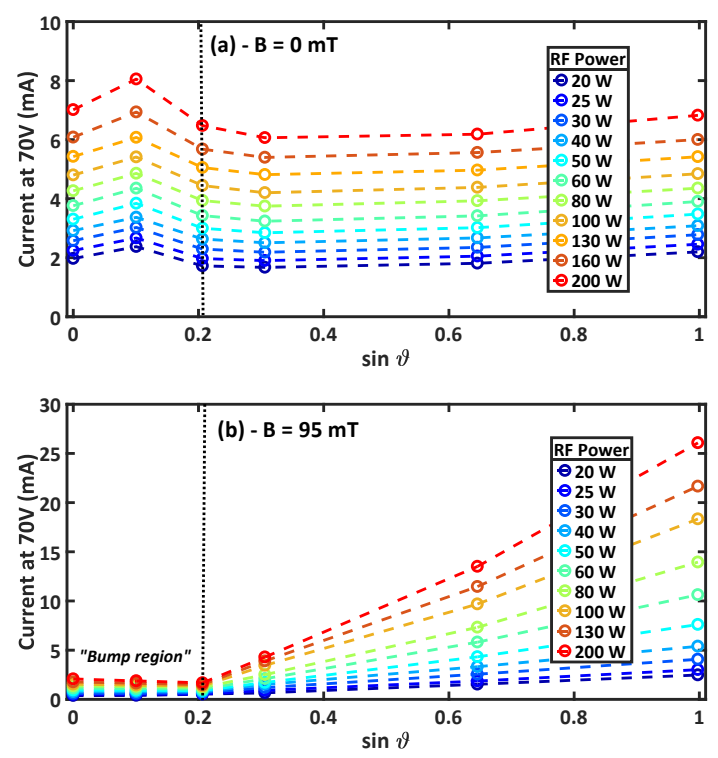

FIG. 8. Evolution of the collected current at $70 \mathrm{~V}$ with the sine of the inclination angle $\vartheta$ without magnetic field (a), and with magnetic field (b) of amplitude $95 \mathrm{mT}, 1.2$ $\mathrm{Pa} \mathrm{He}$. The left region is the "bump region", where a bump is measured $\left(\vartheta \leq 12^{\circ} \Leftrightarrow \sin \vartheta \leq 0.21\right)$. The line is a guide for the eye.
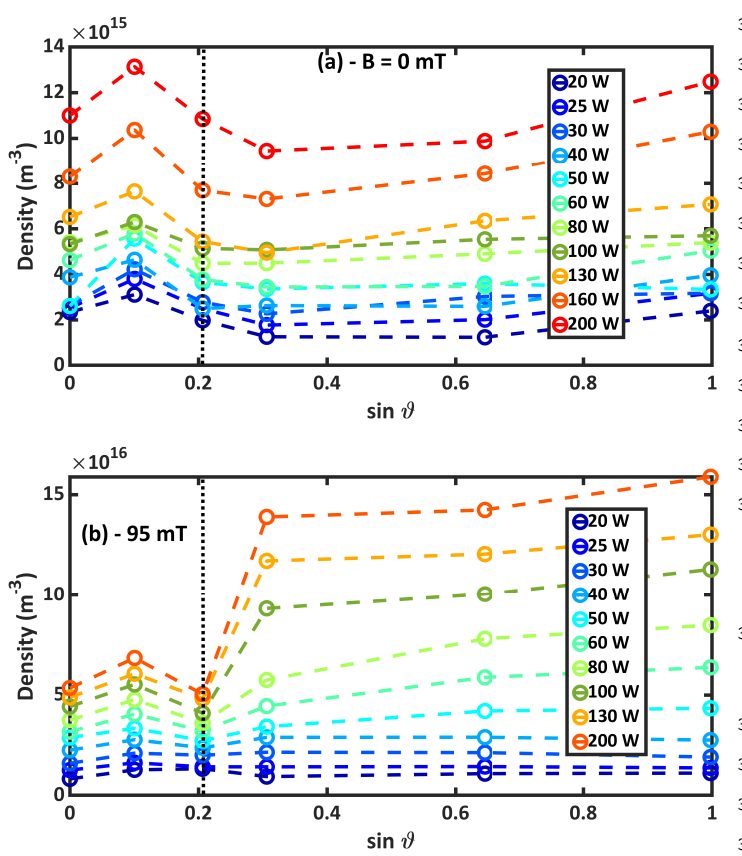

FIG. 9. Evolution of the density measured with the method described in the next section, in same conditions as in figure 8 with magnetic field (a) and without magnetic field (b) of $95 \mathrm{mT}$. As expected, the density is kept approximatively constant in the absence of magnetic field, but we notice a sharp change in the density between the "bump-" and the "no-bump-region" in the presence of magnetic field at higher power. The line is a guide for the eye.

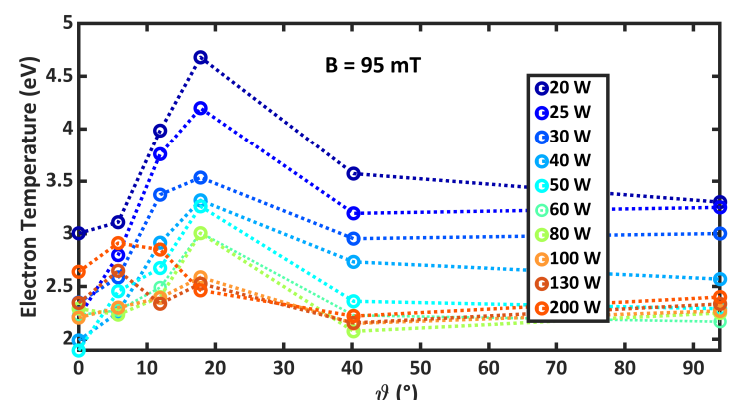

FIG. 10. Evolution of the computed electron temperature (see next section for the used algorithm) with respect to the inclination of the probe at $95 \mathrm{mT}$ magnetic field amplitude. The line is a guide for the eye.

${ }_{318} n_{e} \approx 5 \times 10^{15} \mathrm{~m}^{-3}$ with a bump to $n_{e} \approx 15 \times 10^{15}$ $319 \mathrm{~m}^{-3}$ without a bump at $200 \mathrm{~W}$ RF power). This 320 density difference is enhanced for higher power. 321 However, for lower power the density remains ap322 proximatively constant at all inclinations.

However, the evolution of the electron temper324 ature with respect to the inclination angle (figure 325 10) is impossible to explain straightforwardly. In326 deed the electron flow collected by the probe is the 327 combination of two populations: the parallel and 328 the perpendicular to B flow, having each its own 329 temperature (i.e. $T_{e \|}$ and $T_{e \perp}$ resp.). Our method 330 gives a kind of average of both. Unfortunately, the 331 electron energy distribution function, which could 332 help us to understand the plot, is too noisy to be ex33 ploited (even after some filtering such as Stavitzky 334 Golay, or Fourier analysis). The explanation of this 335 plot is still an opened question for further studies.

Nevertheless, constant end-current in bump ${ }_{337}$ region can also mean there is a surrounding electron 338 sheath assuming the probe potential is higher than 339 the plasma potential, and then the effective collect340 ing radius is higher than the probe radius.

\section{${ }_{341}$ C. Influence of the RF-power}

As shown in the last subsection, increasing RF${ }_{343}$ power also increases the overall density. To track 344 the bump evolution with RF-power regardless to 345 the density change, it is convenient to normalize 346 the $I(V)$ to the end-current value $I(V) / I(70 \mathrm{~V})$. In 347 fig. 11 are depicted the normalized probe character348 istics at $\|\mathbf{B}\|=95 \mathrm{mT}$ for all inclinations and for 349 several input RF-power, fig.11-(a) to (c).

Although the end current is proportional to the 351 collecting surface (which is $\propto \sin \vartheta$ ), the normal352 ization removes this dependence and all angles can 353 be compared. The electron saturation part, directly 354 connected to the sheath extension, is then the same 355 for every angles, as shown in fig.11. In fig.11-(a), 

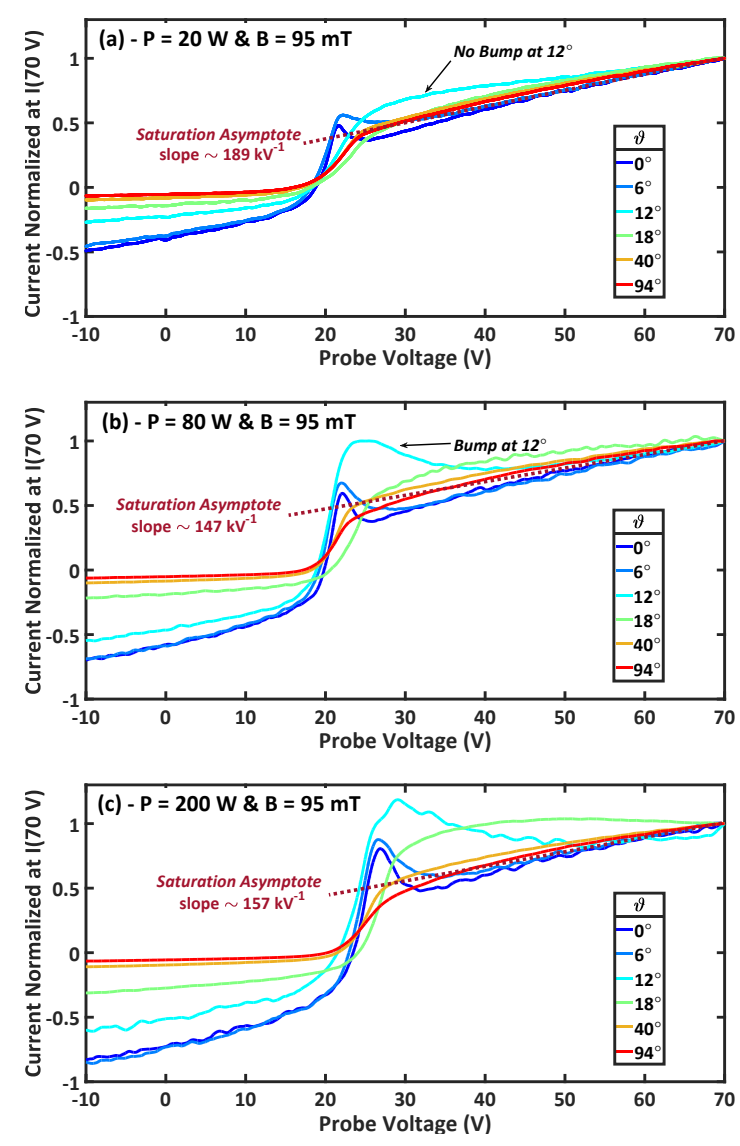

FIG. 11. Normalized $I(V) / I\left(V_{\max }\right)$ characteristics at 95 $\mathrm{mT}$, for every inclination angles, 1.2 $\mathrm{Pa} \mathrm{He}$. RF-power is fixed at (a) $20 \mathrm{~W}$, (b) $80 \mathrm{~W}$ and (c) $200 \mathrm{~W}$. On each graph is also plotted (on dashed lines) the mean saturation linear curve with its slope.

356 for $20 \mathrm{~W}$ there is no bump at $12^{\circ}$, contrary to fig. 11 ${ }_{357}$ (b) for $80 \mathrm{~W}$. The current at the bump position is 358 also larger than the end-current in fig.11-(c). More359 over, the increase of the power increases the ampli360 tude of the bump and its width.

361 One can suppose the existence of perpendicu362 lar (to B) RF currents, pumping the flux tube con363 nected to the probe: this idea is used to derive 364 a fluid model in section IV to recover the bump 365 analytically. In addition, as depicted in fig.8-(b), 366 increasing the power does not increase the end367 current in the "bump region", corroborating the for368 mer assumption. These RF currents, when averaged 369 over one RF period, exhibit a net DC perpendicu370 lar contribution ${ }^{31}$, acting as perpendicular DC cur- 402 371 rents, which have already been investigated in pre372 vious models ${ }^{22,23}$ to explain the depletion and satu373 ration currents of biased flux tubes.

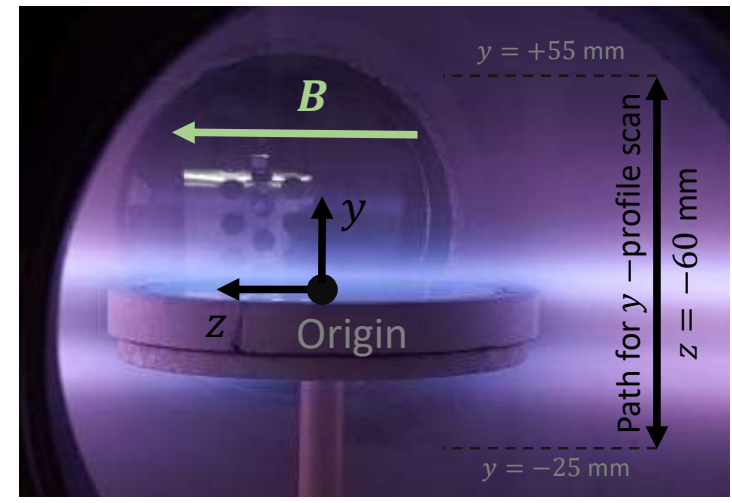

FIG. 12. Photograph from $1.2 \mathrm{~Pa} \mathrm{He}$ pressure plasma around the RF antenna operating at $100 \mathrm{~W}$ with $\|\mathbf{B}\|=80$ $\mathrm{mT}$ magnetic field. The magnetic confinement generates this double player plasma aspect around the probe. Far enough from the antenna the density is homogeneous.

\section{Influence of the probe position}

375 The position of the probe is also an important 376 parameter. It is initially placed relatively far from 377 the antenna to have a homogeneous plasma around 378 the probe. Indeed, near the antenna, the $\mathbf{E} \times \mathbf{B}$ ef379 fect is larger and the plasma denser. That is why, 380 there is a thin plasma layer above, and below the 381 antenna (see photograph in fig.12). Moreover, at ${ }_{32}$ this RF-pulsation ions do not react to the quick po83 tential change near the antenna, whereas electrons ${ }_{84} \mathrm{do}^{32}\left(\omega_{\mathrm{pe}}>\omega_{\mathrm{RF}}>\omega_{\mathrm{pi}}\right)$.

To make sure that the inclination of the probe 386 does not scan different slices of plasma (i.e. that 387 the plasma is homogeneous in a range of $\pm L_{p} \sin \vartheta$ 388 around the probing position in the $y$ direction), mea389 surements along the $y$ axis were performed at fixed $390 z=-60 \mathrm{~mm}$ position and for $\vartheta=0^{\circ}$. Power was 391 fixed at $100 \mathrm{~W}-\mathrm{RF},\|\mathbf{B}\|=80 \mathrm{mT}$ in $1.2 \mathrm{~Pa} \mathrm{He}$ 392 plasma. All characteristics in Fig.13 depicted a 393 bump, where the plotted parameters are the float394 ing potential $\phi_{\text {fll }}$, the bump potential $V_{\text {bump }}$ and the 395 bump current $I_{\text {bump. }}$. Dote suggested the bump po396 tential to be near the plasma one ${ }^{15,19,20}$. According 397 to Dote's assumption and using the combined potential drops in the sheath and the collisionless pre399 sheath $^{1}$, one can write the potential drop between 400 the plasma and the floating probe potential for cold 401 ions $\left(T_{i} / T_{e} \rightarrow 0\right)$ as:

$$
\phi_{p}-\phi_{\mathrm{fl} .}=\frac{T_{e}}{2 e} \ln \left[\frac{m_{i}}{2 \pi m_{e}}\right]+\frac{T_{e}}{2 e}=4.03 \times T_{e},
$$

with $T_{e}$ in eV. For all previous measurements at ${ }_{403}(z=-60 \mathrm{~mm}, y=40 \mathrm{~mm})$, using the approxima404 tion $\phi_{p} \approx V_{\text {bump }}$ gives $T_{e} \approx 1.30 \mathrm{eV}$ (which is a typ406 ical value in ALINE magnetized, plasma ${ }^{26,27}$ ). 


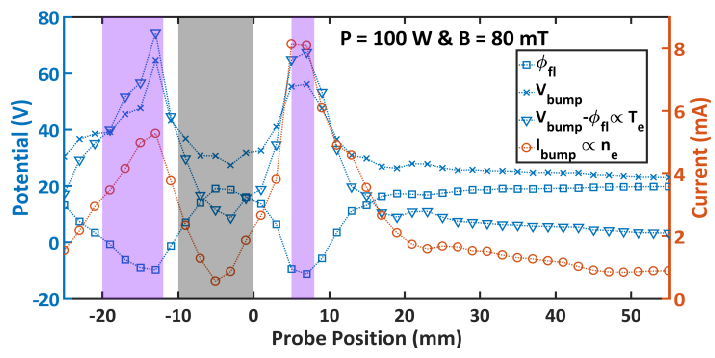

FIG. 13. Evolution of measured parameters (floatting potential $\phi_{\text {fl. }}$, bump potential $V_{\text {bump }}$ and bump current $\left.I_{\text {bump }}\right)$ along the $y$ axis at $z=-60 \mathrm{~mm}, 100 \mathrm{~W}-\mathrm{RF}, 80$ $\mathrm{mT}$ and $1.2 \mathrm{~Pa}$ (see double arrow $\leftrightarrow$ in fig.12). The gray region represents the region where the probe faces the antenna (antenna extension is $z \in[-40,40] \mathrm{mm}$ and $y \in[-10,0] \mathrm{mm})$, the purple regions represent the denser plasma region (see photograph in fig.12). For comparison $T_{e} \propto V_{\text {bump }}-\phi_{\text {fll. }}$ is also plotted

407 The tilting of the probe does not scan "different 408 slices" of plasma and different inclination angles 409 can be compared as shown in fig. $13: 1 \mathrm{~cm}$ around ${ }_{410}$ the $y=40 \mathrm{~mm}$ position, all cited parameters are ${ }_{411}$ almost constant. Therefore, the homogeneity hy412 pothesis (almost constant $T_{e}$ and $n_{e}$ in the probing 413 area) can be applied in the experimental conditions. ${ }_{414}$ Finally, this last figure also highlights the fact that 415 current and temperature (as defined in eq. 7) in${ }_{416}$ creases by a factor of $\sim 7$ in the bright regions (see ${ }_{417}$ photograph depicted in fig.12), near the antenna.

\section{${ }_{418} \mathrm{E}$. Influence of the pressure}

419 For a magnetic field of $80 \mathrm{mT}$, and an input 420 power of $80 \mathrm{~W}-\mathrm{RF}$, measurements were performed 421 with a probe parallel to the field line $\left(\vartheta=0^{\circ}\right)$ in a ${ }_{422}$ He pressure range from 1.2 to $40 \mathrm{~Pa}$. All character423 istics are plotted Fig. 14.

424 When pressure increases, the bump gets narrower 425 and its amplitude diminishes. Above 9.32 $\mathrm{Pa}$, the 426 bumps disappear and the $I(V)$ characteristic turns ${ }_{427}$ into an asymmetric double probe one.

${ }_{428}$ That's why one can separate the pressure range 429 in 2 regimes :

${ }_{430}$ The low collisionnal regime from $1 \mathrm{~Pa}$ to 10 ${ }_{431} \mathrm{~Pa}$. At these pressures the electron-neutral col${ }_{432}$ lision frequency $v_{\text {col. }}^{e N}$ is lower than the electron 433 plasma frequency $v_{p e}$, and lower than the electron ${ }_{434}$ cyclotron frequency $v_{c e}$ (see table I) and of the same 435 order than the RF frequency. For example at $1 \mathrm{~Pa}$ ${ }_{436} v_{\text {col. }}^{e N} \approx 17 \mathrm{MHz}^{29}$. In the same way the ion-neutral ${ }_{437}$ collision frequency $v_{\text {col. }}^{i N}$ is much lower than the ion ${ }_{438}$ plasma frequency $v_{p i}$, and lower than the ion cy439 clotron frequency $v_{c i}$ up to $4 \mathrm{~Pa}$ so that ions are 440 considered as magnetized in the first half of the low ${ }_{441}$ collisionnal pressure range. In this range the classi-

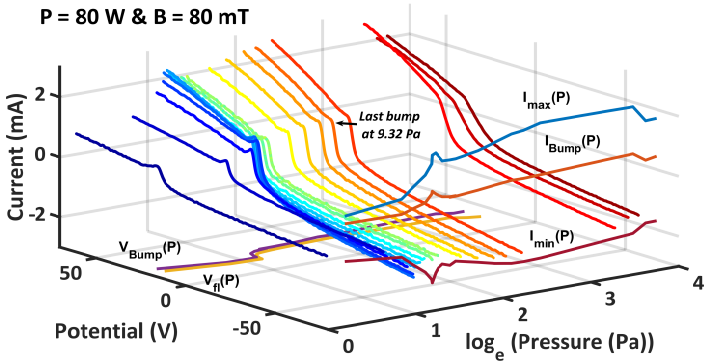

FIG. 14. Tridimensionnal representation of the $I(V)$ characteristics in all considered He pressures from 1.2 to 40 $\mathrm{Pa}$ for $80 \mathrm{~W}$-RF power and $\|\mathbf{B}\|=80 \mathrm{mT}$. In the $V=80$ $\mathrm{V}$ plane are plotted the end currents at $\pm 70 \mathrm{~V}$ and the bump current. In the $I=-3 \mathrm{~mA}$ plane are plotted the floating and the bump potentials. Last bump is measured at $9.32 \mathrm{~Pa}$. If no bump is measured, $I_{\text {bump }}$ corresponds to the point where $\mathrm{d} I / \mathrm{d} V=\max (\mathrm{d} I / \mathrm{d} V)$, i.e. the current at "classical" plasma potential.

442 cal perpendicular diffusion falls down and perpen443 dicular currents are able to deplete strongly the flux 444 tube while the typical scale length of these current 445 is higher than the radius of the probe, which is the ${ }_{446}$ case here because $\rho_{c i} \gg r_{p}$. In a quiet plasma, as ${ }_{447}$ we have in ALINE, such a behaviour can be seen, 448 while in Tokamak edge plasma anomalous transport 449 can still prevent the biased flux tubes to deplete.

${ }_{450}$ In the collisionnal regime $(P>10 \mathrm{~Pa}), v_{\text {col. }}^{e N}$ re451 mains lower than $v_{c e}$ and $v_{p e}$, but much higher than 452 the RF frequency. RF electron current are then low453 ered by collisions. And ions are no more magne${ }_{454}$ tized because $v_{\text {col. }}^{i N}>v_{c i}$, which favours their per455 pendicular diffusion while ion perpendicular cur456 rent are lowered in the same time, filling the lack 457 of density caused by the probe collection and can458 celling the bump on the characteristics. For the 459 highest pressures, the flux tube for ions disappears ${ }_{460}$ and the $I(V)$ looks like an unmagnetized one ${ }^{14}$.

${ }_{461}$ In the intermediate case of partially magnetized 462 ions, the $I(V)$ looks like a double symmetric probe ${ }_{463}$ characteristics. The electron saturation current col464 lected by the probe depends also on the competition 465 between perpendicular DC and RF currents and the 466 cross diffusion of ions due to collisions.

Another remarkable result depicted in fig.14 is 468 that, when the pressure is increased by a factor of ${ }_{469} 40$, the maximal current at probe position only in470 creases by a factor 2 . This behaviour denotes a good 471 confinement of the plasma around the antenna by 472 the magnetic field. Indeed, increasing the pressure 473 brightens the plasma shown in fig.12; however out474 side this region the plasma remains more or less the 475 same. The only thing that changes is the collision 476 rate with neutrals at higher density. 


\section{IV. THEORETICAL APPROACHS}

478 In the first part of this section, we provide a quan479 titative comparison of three different methods used 480 to extract both $n_{e}$ and $T_{e}$ from bumped character481 istics. In a second part, we show by using a fluid ${ }_{482}$ model that, the bump in the $I(V)$ curves in a pres483 ence of a magnetic field, can be explained by mean 484 of density depletion within the tube flux connected 485 to the probe and to the opposite wall of the reactor.

\section{${ }_{486}$ A. Density and temperature data processing}

487 Extracting electron density and temperature from ${ }_{488} I(V)$ characteristics is far from simple. But if 489 the measurements are done in the presence of 490 a magnetic field, the exploitation are even more 491 difficult. The challenge lies on the presence of 492 the bump, whose existence, shape, location and 493 amplitude depend on several plasma parameters ${ }_{494}(\|\mathbf{B}\|, \vartheta$, Pwr., $y, p)$ (see sections III A to III E).

495 The first problem with bumped characteristics is 496 the uncertainty on the position of the plasma po497 tential. It is usually found by assuming that, at the 498 plasma potential $V=\phi_{p}, \mathrm{~d} I /\left.\mathrm{d} V\right|_{\phi_{p}}=\max (\mathrm{d} I / \mathrm{d} V)$, 499 which is equivalent to $\mathrm{d}^{2} I /\left.\mathrm{d} V^{2}\right|_{\phi_{p}}=0^{2,3}$ (this is 500 called the "classical method" in the following). An501 other method based on the intersection of the linear 502 fits of the exponential part and the electron satura503 tion one has also been suggested and used in a pre504 vious study ${ }^{17}$. It was finally suggested that, in the 505 context of bumped characteristics, the bump poten506 tial is at the plasma potential ${ }^{15,19}$. Thus, three meth507 ods are available, in order to determine the plasma 508 potential and we propose to compare them, for dif509 ferent inclinations, in a single $100 \mathrm{~W}$-RF plasma, 510 with $\|\mathbf{B}\|=80 \mathrm{mT}$ and $p=1.2 \mathrm{~Pa}$, whose charac511 teristics are depicted in fig.15-(a).

${ }_{512}$ We assume that the best method is the one which 513 would exhibit the lowest deviation of the plasma pa514 rameters with respect to $\vartheta$. We suppose indeed that 515 the probed plasma slice is the same for all inclina516 tions.

517 In the context of the "intersection method" 518 we linearised the exponential growth as $I(V) \approx$ ${ }_{519} a_{\text {exp. }} V+b_{\text {exp., }}$, and fitted the electron saturation cur520 rent with the formula:

$$
I_{e}(V) \approx a_{\text {sat. }} V+b_{\text {sat. }}+c_{\text {sat. }} \sqrt{V} .
$$

${ }_{521}$ with the $\sqrt{V}$ term similar to one of the OML ap${ }_{522}$ proach, which gives a relatively good fit with ex523 perimental curves. This equation is only able to fit 524 the saturation part, i.e. the end of the $I(V)$ - far ${ }_{525}$ from the bump potential range. Only the last 20 526 volts of each $I(V)$ were used for the fitting, see fig. 527 15-(a).
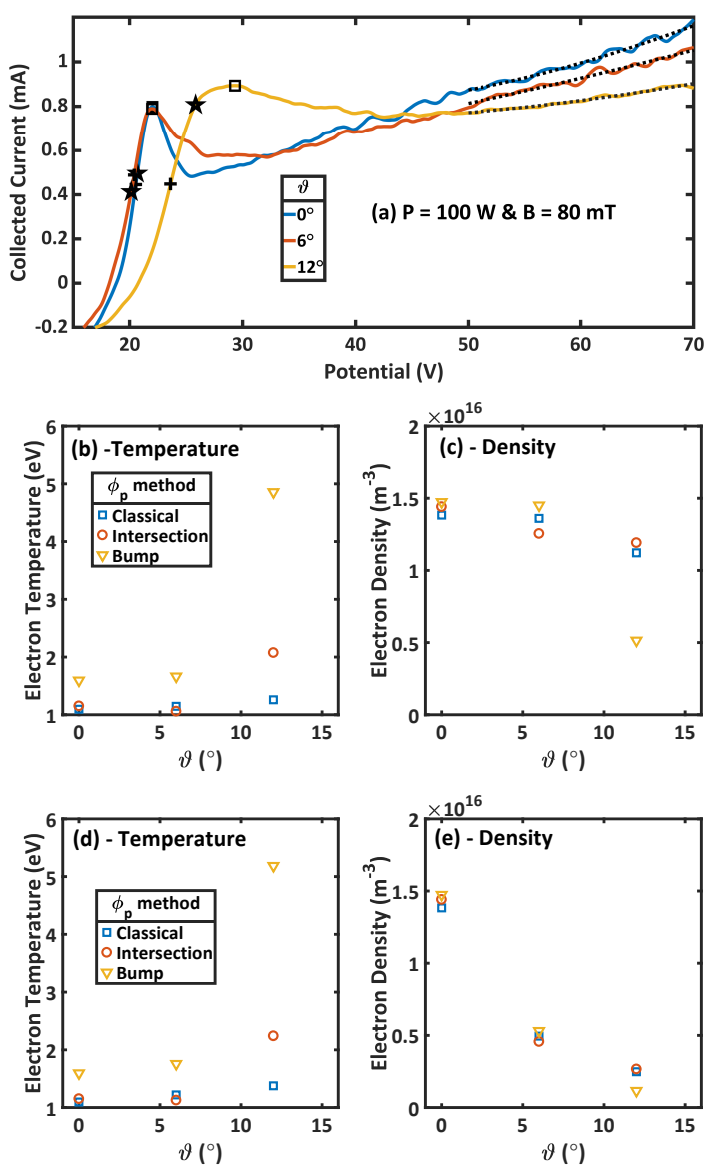

FIG. 15. Results of the electron temperature and density calculation on bumped characteristics with the described iterative model : (a) $I(V)$ of plasma at $100 \mathrm{~W}-\mathrm{RF}$, $80 \mathrm{mT}$ and 1.2 $\mathrm{Pa}$ for different probe inclinations and all methods are represented for the position of $\phi_{p}(+$ classical, $\star$ intersection, $\square$ bump). The dashed line is the fit of the electron saturation current with respect to equation (8) - (b) and (c) $T_{e}$ and $n_{e}$ against inclination angle with collecting surface correction - (d) and (e) $T_{e}$ and $n_{e}$ against inclination angle without collecting surface correction, $S_{\text {coll. }}=S_{\text {probe }}$ ( $T_{e}$ remains the same though).

${ }_{528}$ We used an iterative method, in order to deter${ }_{529}$ mine both $n_{e}$ and $T_{e}$ with the plasma potential $\phi_{p}$, 530 the current at plasma potential $I_{p}$, floating poten${ }_{531}$ tial $\phi_{\mathrm{fl}}$, magnetic field and probe inclination as 532 input parameters. First, a raw approximation of 533 electron temperature is done, supposing $I \sim I_{e} \propto$ ${ }_{534} \exp \left(e V / k_{B} T_{e}\right)$ for $V \leq \phi_{p}$ in the exponential part. 535 Applying a linear fit on $\ln I(V)$ one will find a first 536 value of $T_{e}$. From now one starts the iterative loops: 537 this electron temperature value allows a computa538 tion of a gross value of $n_{e}$ since, at plasma potential, ${ }_{539} I_{p}=e n_{e}\langle v\rangle S_{e} / 4$. The value of $S_{e}$ is not the probe 540 surface, even at plasma potential (where there is no ${ }_{541}$ sheath), because of the cyclotron motion. That is 542 why it is assumed that the collecting surface is the 
${ }_{543}$ probe surface facing $\mathbf{B}$ plus a layer thick of $N_{\text {elr. }} \rho_{c e}$ 544 (i.e. some Larmor radii $-N_{\text {elr. }}$ being the number of 545 electron Larmor radii connected to the probe) :

$$
\begin{aligned}
S_{e}=\pi & \cos \vartheta \times\left(r_{p}+N_{\text {elr. }} \rho_{c e}\right)^{2} \\
& +\pi L_{p} \sin \vartheta \times\left(r_{p}+N_{\text {elr. }} \rho_{c e}\right)
\end{aligned}
$$

546 by replacing $r_{p} \rightarrow r_{p}+N_{\text {elr. }} \rho_{c e}$ in eq.(6). It is as547 sumed that this equation takes into account the per548 pendicular motion of electrons along the magnetic 549 field lines connected to the probe.

${ }_{550}$ With $T_{e}$ and $n_{e}$, it is possible to compute the elec${ }_{551}$ tron Debye length $\lambda_{\text {De }}$ and the ion sheath thickness 552 using the Child-Langmuir law (since $\rho_{c i} \gg r_{p} \sim \rho_{c e}$ 553 and that Zhu's corrections ${ }^{33}$ for cylindrical geome554 try only bring minor changes in opposition to its 555 complexity), knowing,

$$
\ell_{\mathrm{CL}}=\frac{\sqrt{2}}{3} \lambda_{\mathrm{De}}\left(2 e \frac{\left|\phi_{p}-V\right|}{k_{B} T_{e}}\right)^{3 / 4}
$$

${ }_{556}$ for $V \leq \phi_{p}$. Since ions are supposed unmagnetized, 557 the collecting area for ions is

$$
S_{i}=\pi\left(r_{p}+\ell_{\mathrm{CL}}\right)^{2}+2 \pi L_{p}\left(r_{p}+\ell_{\mathrm{CL}}\right) .
$$

558 It is then possible to compute the ion current 559 for $V \leq \phi_{p}$, using the Bohm flux formula, $I_{i}=$ $5600.61 \times e n_{e} c_{S} S_{i}$. So, the updated electron current ${ }_{561} I_{e}=I(V)-I_{i}$ can be calculated. Taking again the ${ }_{562} \log$-scale of this new electron current gives a new ${ }_{563}$ more accurate value of $T_{e}$. The loop starts over 564 again, and ends if temperature values converge (i.e. ${ }_{565}\left|T_{e}^{\text {new }}-T_{e}^{\text {old }}\right| \leq \varepsilon, \varepsilon$ being given by the user).

${ }_{566}$ Equations giving $S_{e}$ and $S_{i}$ (eqs. (9) and (11) 567 resp.) take into account the sheath extension for 568 magnetized electrons and unmagnetized ions. To 569 take into account the inclination of the probe, and 570 find reliable plasma parameters, one should also 571 multiply the total current by a geometric factor of ${ }_{572} \pi r_{p}^{2} / S_{\text {face }}$ from eq.(6) giving a dimensionless fac573 tor of $1 /\left(\cos \vartheta+\left[L_{p} / r_{p}\right] \times \sin \vartheta\right)$. This allows the 574 recovering of the same amplitude for all bumped ${ }_{575} I(V)$. The extracted values of $n_{e}$ and $T_{e}$ are plot576 ted in fig.15-(b) and (c) using this correction, and 577 plotted in fig.15-(d) and (e) without the correc578 tion ( $n_{e}$ strongly decreases with the angle). From ${ }_{579}$ Fig. 15-(b) and (c) it is clear that the classical $\phi_{p^{-}}$ 580 determination method gives the more reliable val${ }_{581}$ ues of temperature and density (the deviation of $T_{e}$ ${ }_{582}$ values between each inclination is negligible com${ }_{583}$ pared to other methods). We have then $T_{e} \approx 1.2 \mathrm{eV}$ ${ }_{584}$ and $n_{e} \approx 1.3 \times 10^{16} \mathrm{~m}^{-3}$. Since the OML model 585 remains valid in $\mathrm{RF}$-plasmas ${ }^{34}$, and that ions are 586 unmagnetized, we extracted $n_{i}^{\mathrm{OML}}=1.74 \times 10^{17}$ ${ }_{587} \mathrm{~m}^{-3}$ (which is within the typical errorbar for OML ${ }_{588}$ model) and $T_{e}^{\mathrm{OML}}=2.69 \mathrm{eV}$, which are overesti589 mated compared to the previous method.

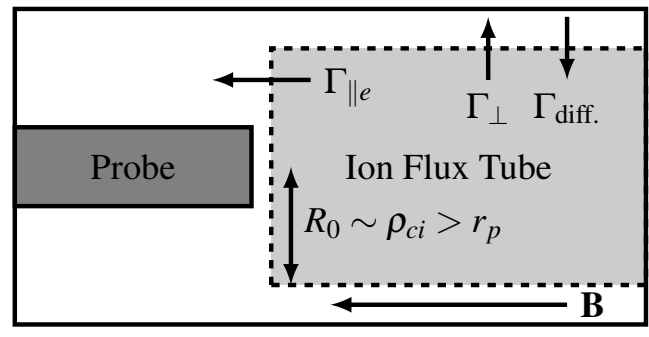

FIG. 16. Sketch of the fluid model. The flux tube is delimited by the dashed line. The inclination $\vartheta$ is $0^{\circ}$.

By comparison, in the absence of magnetic field, 591 the bump method to find the plasma potential makes ${ }_{592}$ no sense (since there is no bump) and both classi${ }_{593} \mathrm{Cal}$ and intersection methods are alike and give the 594 same value of the plasma potential. Therefore the 595 self-consistent algorithm gives an electron density 596 of the order of $5.32 \times 10^{15} \mathrm{~m}^{-3}$ and an electron tem${ }_{597}$ perature of $3.47 \mathrm{eV}$ (for the same discharge param598 eters as with $\|\mathbf{B}\| \neq 0)$.

\section{${ }_{599}$ B. Fluid model approach}

As suggested by Mihaila and Rozhansky ${ }^{16,23}$, the 601 bump on $I(V)$ characteristics could be induced by ${ }_{602}$ density depletion within the flux tube.

The cylindrical flux tube connected from the 604 probe to the reactor's wall is filled by electrons us605 ing a single channel, which is the lateral area of 606 the cylinder. Due to magnetic confinement and for 607 grazing incidences, the perpendicular current arises 608 thanks to collisions with neutrals. During a $I(V)$ 609 measurement for $V>\phi_{p}$, one pumps the electrons 610 inside the flux tube, which makes the local elec611 tron density decreases. If the pumped electron cur${ }_{612}$ rent is larger than the refill perpendicular one, then ${ }_{613}$ the collected current at the probe decreases with ${ }_{614}$ phi (the bump origin). But when the probe poten615 tial is increased further, the sheath extent around it ${ }_{616}$ also increases, which artificially makes the cylindri${ }_{617}$ cal flux tube diameter wider. Consequently, when ${ }_{618} V \gg \phi_{p}$, the electron perpendicular current com619 pensate the pumped one and the collected current 620 increases again. Now for larger incidences, the per${ }_{621}$ pendicular current always overcomes the pumped 622 one, which explains the experimentally observed 623 disappearance of the bump for $\vartheta>12^{\circ}$.

${ }_{624}$ In the meantime, there is another mechanism in625 volving mainly ions: it is the plasma pumping via ${ }_{626}$ perpendicular ion current due to the positive bias${ }_{627}$ ing of the flux tube with respect to the surround${ }_{628}$ ing plasma potential. This mechanism has already ${ }_{629}$ been invoked to explain the early electron satura${ }_{630}$ tion of the $\mathrm{I}(\mathrm{V})$ characteristics in the case of planar 
${ }_{631}$ probe $^{23}$ in magnetized plasmas. The typical scale ${ }_{632}$ length of these perpendicular ion currents is the ion ${ }_{633}$ gyroradius. To explain the bump, this mechanism ${ }_{634}$ can be divided in three regimes occurring when the 635 probe potential overcomes the plasma potential:

1. When the transverse (perpendicular to $\mathbf{B}$ ) ion current is lower than the electron saturation current collected by the probe, the space charge of the sheath is electropositive and consequently the flux tube potential "follows" the probe potential. The density depletion can first appear in that regime.

2. When the transverse ion current is exactly equal to the electron saturation current collected by the probe, the sheath between the probe and the flux tube disappears and the collected current can decrease because the flux tube density decreases with the probe potential.

3. Finally when the transverse ion current is higher than the electron saturation current collected by the probe, electrons must be accelerated in the sheath to balance the ion current and thus the sheath drop is reversed. The sheath space charge becomes electronegative and the flux tube potential tends to saturate compared to the probe potential. This regime accounts for long and thin flux tube.

Nevertheless, plasma diffusion is more and more ${ }_{660}$ efficient as the flux tube is widening. So in the third ${ }_{661}$ regime, with the saturation of the flux tube poten${ }_{662}$ tial, the pumping also saturates and the density de${ }_{663}$ pletion can be cancelled resulting in a classical in${ }_{664}$ crease of the current in the last part of the $I(V)$ char665 acteristics (beyond the bump).

${ }_{666}$ Finally there is an optimum point for which the ${ }_{667}$ pumping is maximum compared to cross diffusion, 668 and this is at this working point the bump appears to ${ }_{669}$ be the higher because of the strong negative slope 670 just following the maximum of the bump. Actu671 ally, the bump does not mean there is an increase of 672 current compared to an $I(V)$ characteristics with no 673 bump, on the contrary it means a decrease of cur674 rent.

675 The complexity of the phenomenon can only be 676 explained by a mass and current conservation tak677 ing into account the growing of the flux tube radius 678 with the potential.

\section{The model:}

680 Rozhansky et al. ${ }^{23}$ showed that the ion flux tube ${ }_{681}$ has a characteristic radius of $R_{0} \sim \rho_{c i}$, and a length ${ }_{682} L$ (this ion flux tube connected to the probe also
${ }_{683}$ contains the electron flux tube of radius $\rho_{c e} \ll \rho_{c i}$ ). ${ }_{684}$ Due to their cyclotron motion, electrons are trapped 685 in both ion and electron tubes and can only leave 686 them through the ends, producing a parallel net cur${ }_{687}$ rent of $J_{e \text {,sat. }} \times \pi R_{0}^{2}$. To ensure current and quasi${ }_{688}$ neutrality conservation in the ion tube, there must

689 be a perpendicular ion flux through the cylindrical ${ }_{690}$ surface so that, $J_{i \text {,sat. }} \times 2 \pi R_{0} L \approx J_{e \text {,sat. }} \times \pi R_{0}^{2}$. In 691 this regime, where the perpendicular current can 692 be higher than the electron saturation current on 693 the probe, the potential gap can reverse in front ${ }_{694}$ of the probe (electronegative sheath) accelerating 695 electrons and repelling ions. Thus, one can ne${ }_{696}$ glect the parallel ion flux on the probe side (in the ${ }_{697}$ case of an electropositive sheath, the ion current on ${ }_{698}$ the probe surface can also be neglected compared 699 to electron current, still assuming that the electron 700 current is close to its saturation value).

In the following we use current continuity for ${ }_{702}$ ions in order to obtain a first order ODE that gives 703 the density of the flux tube with respect to the probe 04 potential. Using Laframboise's theory, this tube 05 density (or "local plasma density") gives the elec706 tron fraction that will be collected by the probe re707 garding its potential $V$. An analytic expression of 08 the collected current can be then provided.

As shown in the last sections, the pumping is 10 enhanced by perpendicular (to B) RF and DC currents $^{21,22}$. But periodic RF current can be re712 duced to an averaged DC over one a period. That is 713 why the model is steady state, and only DC quan714 tities are considered. Finally, to prevent the tube 715 density to drop to zero, we assume the presence of 716 a source term $S_{0}$, so that,

$$
\iiint_{\text {tube }} S_{0} \mathrm{~d} \tau=2 \times \pi R_{0}^{2} \times \frac{1}{2} n_{0}\left\langle v_{e}\right\rangle
$$

717 where $n_{0}$ is the bulk plasma density (outside the ion ${ }_{718}$ flux tube region) and $n_{t}$, the ion flux tube density ${ }_{719}\left(n_{0} \geq n_{t}\right)$. This term fills the tube at the same rate 720 electrons leave it from both ends (which is an over721 estimation of the "real" $S_{0}$ source term).

From the stationary ion continuity equation, we ${ }_{723}$ have $\boldsymbol{\nabla} \cdot \boldsymbol{\Gamma}_{i} \sim \nabla \cdot \boldsymbol{\Gamma}_{i, \perp}=S_{0}$. Perpendicular ion flux 724 is separated in two parts : lateral mobility $-\mu_{i} n_{t} \nabla \phi$ 725 and the diffusion flux $-D_{\perp} \nabla n_{t}$. Integration of all 726 ion fluxes through the whole tube using Gauss's law 727 gives:

$$
\frac{n_{0}\left\langle v_{e}\right\rangle}{2 L} R_{0}=-\left(\left.D_{\perp} \frac{\partial n_{t}}{\partial r}\right|_{R_{0}}+n_{t} \mu_{i} \times\left.\frac{\partial \phi}{\partial r}\right|_{R_{0}}\right)
$$

${ }_{728}$ In the presence of a strong radial electric field (and ${ }_{729}$ especially in a cold plasma), the ion drift veloc730 ity is larger than the thermal velocity, thus $\rho_{c i}=$ ${ }_{731} v_{\perp} / \omega_{c i}=\left(v_{\text {drift }}^{2}+v_{i, \text { Th. }}^{2}\right)^{1 / 2} / \omega_{c i} \sim\left|v_{\text {drift }}\right| / \omega_{c i}=$ ${ }_{732}-\partial_{r} \phi / B \omega_{c i}$ (all at $\left.R_{0}\right)$. Recalling that $R_{0} \sim \rho_{c i}$, 
733 equation (13) rewrites as,

$$
\frac{n_{0}\left\langle v_{e}\right\rangle}{2 L B \omega_{c i}} \times\left.\frac{\partial \phi}{\partial r}\right|_{R_{0}}=\left(\left.D_{\perp} \frac{\partial n_{t}}{\partial r}\right|_{R_{0}}+n_{t} \mu_{i} \times\left.\frac{\partial \phi}{\partial r}\right|_{R_{0}}\right)
$$

734 Now using the chain rule, $\partial n_{t} /\left.\partial r\right|_{R_{0}}=\partial n_{t} / \partial \phi \times$ ${ }_{735} \partial \phi /\left.\partial r\right|_{R_{0}}$, one will get the following first order ${ }_{736} \mathrm{ODE}$ at the radius $r=R_{0}$ :

$$
\frac{\partial n_{t}}{\partial \phi}=-\frac{\mu_{i}}{D_{\perp}} n_{t}+\frac{n_{0}\left\langle v_{e}\right\rangle}{2 \omega_{c i} B L D_{\perp}}
$$

${ }_{737}$ The perpendicular mobility can also be written as a 738 conductivity depending on the current nature (col739 lision, inertial, viscosity, anomalous,...). With the 740 initial condition of $n_{t}\left(\phi=\phi_{p}\right)=n_{e}$ since there is 741 no sheath nor spatial potential variation at plasma 742 potential, one will get :

$$
n_{t}(V)=n_{\infty}+\left(n_{0}-n_{\infty}\right) \exp \left[\frac{\mu_{i}}{D_{\perp}}\left(V-\phi_{p}\right)\right],
$$

${ }_{743}$ where $V$ is the probe potential and $n_{\infty}=n_{0} \times$ ${ }_{744}\left\langle v_{e}\right\rangle / 2 \mu_{i} B \omega_{c i} L$. Here we assumed that the flux tube 745 potential equals the probe one. Although generally, ${ }_{746} \phi_{t}=f(V) \geq V>\phi_{p}$.

${ }_{747}$ Equation 16 exhibits an exponential decay of the 748 density with V. This strong depletion of the flux 749 tube as soon as the biased potential of the tube 750 is higher than the surrounding plasma potential is 751 needed to see the bump rising. For lower decay ${ }_{752}$ (for ex. $\sim 1 /\left(V-\phi_{p}\right)$ or $\sim 1 /\left(V-\phi_{p}\right)^{2}$ ) the bump 753 does not appear because of the expansion of the 754 sheath which increases the lateral surface of the flux 755 tube and hence the total perpendicular current more 756 rapidly that the density is depleted. This also ex757 plains why at higher probe potential value, when 758 the exponential decay saturates, the current rises up 759 again due to sheath expansion. Actually there is a 760 competition between the diffusion $D_{\perp}$ accross the 761 lateral surface of the tube versus the perpendicular ${ }_{762}$ current due to ion mobility $\mu_{i}$ as it can be seen in 763 equation 16.

${ }_{764}$ Next to fit the sheath expansion above $V_{p}$ in a 765 magnetic field parallel to the probe, one uses the 766 Laframboise $^{9}$ model which showed that the portion

767 of plasma density actually touching a probe and ${ }_{803}$ V. CONCLUSION 768 thus collected, $n_{\mathrm{eff}}$, is given by the relation,

$$
n_{\text {eff. }}(\xi)=\frac{2 n_{t}(\xi)}{\sqrt{\pi}}\left[\sqrt{\xi}+\frac{\sqrt{\pi}}{2} e^{\xi} \operatorname{erfc} \sqrt{\xi}\right]
$$
${ }_{769}$ for $\xi=e\left(V-\phi_{p}\right) / k_{B} T_{e}$. Finally, the collected cur770 rent on the probe is simply given by

$$
I_{e}(V)=\frac{1}{2} e n_{\text {eff. }}(V) \times\left\langle v_{e}\right\rangle S_{e}
$$

771 where $S_{e}$ is given by eq.(9), and the number of elec772 tron Larmor radii $\left(N_{\text {elr. }}\right)$ is given as fitting parame773 ter.

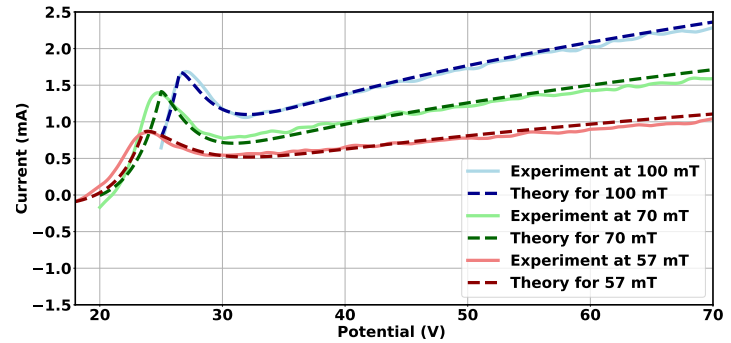

FIG. 17. Comparaison of the 1D fluid model with the experiment. Probe had $\vartheta=0^{\circ}$ inclination angle in 200 $\mathrm{W}-\mathrm{RF}$ plasma at $1.2 \mathrm{~Pa}$ for several $\|\mathbf{B}\|$.

This model is compared with the experimental 775 data in figure 17 for a magnetic field of 57, 70 and $776100 \mathrm{mT}$ in a $200 \mathrm{~W}-\mathrm{RF}$ and $1.2 \mathrm{~Pa}$ Helium plasma 777 (the probe was parallel to $\mathbf{B}$ ). For $V<\phi_{p}$ the expo778 nential $J_{e, \text { sat. }} \times \exp \left(e\left(V-\phi_{p}\right) / k_{B} T_{e}\right) S_{e}$ part of the 779 electronic current is considered.

The number of Larmor radii, $N_{\text {elr. }}$ goes from 0.1 781 to 5 with increasing $\|\mathbf{B}\|$ in Eq. (9). The limit den782 sity in the flux tube, $n_{\infty}$ is close to $n_{0} / 10$ : that 783 means that the measurement heavily depletes the 784 magnetic flux tube. Moreover, the model suggests 785 that the plasma potential is on the top of the bump 786 as proposed by Dote and Mihaila : the pumping 787 mechanism starts when $V>\phi_{p}$ according to the 788 theory. Since electrons are way much mobile than 789 ions along $B$, as soon as the probe potential is above 790 the plasma potential, electrons of the flux tube are 791 flushed towards the probe. Moreover, as pointed out 792 by eq.(3), the flux tube itself has its own potential (slightly above the bulk plasma potential) since it is 794 connected to the probe and thus somehow biased.

Finally, according to this theory, the prior param796 eter is actually the probe surface facing the mag797 netic field lines (i.e. the width of the magnetic flux 798 tube). Therefore, a bump could appear on a plane ${ }_{799}$ probe characteristics or a spherical probe character800 istics as well, if the facing surface is small enough 801 corresponding more or less to a disk surface having ${ }_{802}$ a radius of the order of $\rho_{c e}$.

804 Langmuir probe measurements in the presence of 805 a magnetic field are of a paramount importance in 806 plasma physics. Understanding and exploiting $I(V)$ 807 characteristics from a cylindrical Langmuir probe 808 in such conditions is difficult, especially due to the 809 presence of a bump in the curves for grazing inci810 dences of the cylindrical probe with respect to the 811 magnetic field lines. In this paper, the evolution of 812 the $I(V)$ characteristics with respect to several dis813 charge parameters (magnetic field amplitude, probe 814 inclination, and pressure) was studied, in order to 
815 provide a better understanding of cylindrical Lang816 muir probe measurements in magnetized plasmas.

${ }_{817}$ We showed that the presence of the magnetic ${ }_{818}$ field changes the general shape of the $I(V)$ curves, 819 because of the breaking-up of the plasma isotropy: 820 the particles are not collected by the probe from all ${ }_{821}$ possible directions anymore but from a flux tube, 822 connected to it from one end, and to the reactor's 823 wall to the other. That is why the general shape 824 of the characteristics tends to an asymmetric dou825 ble probe (or tanh-shaped) one. We also showed 826 that for grazing incidences of the probe with re${ }_{827}$ spect to $B$, a bump arises between the exponential ${ }_{828}$ part and the electron saturation current one. The ${ }_{829}$ bump vanishes as the probe inclination is increased, 830 or if the magnetic field amplitude is reduced. It ${ }_{831}$ is also dependent on collisional processes, because ${ }_{832}$ its amplitude decreases, when the gas pressure in833 creases. Finally, increasing the RF-power at the an834 tenna heightens the bump amplitude, and can even 835 make one appearing on the characteristics.

${ }_{836}$ We argued that a probe measurement pumps 837 electrons from their flux tube while ions are ex${ }_{838}$ pelled in the perpendicular direction (the electron 839 current is mainly parallel to magnetic field lines). 840 This density depletion as soon as probe potential ${ }_{841} V$ overcomes the plasma one $\phi_{p}$ (i.e. as the probe ${ }_{842}$ starts to attract electrons) can explain the presence 843 of the bump. This hypothesis is strengthened by 844 the pressure effects on the probe measurements: 845 increasing the gas pressure (thus increasing colli${ }_{846}$ sions and therefore, perpendicular diffusion fluxes), ${ }_{847}$ makes the bump vanish. By using a fluid model, 848 we corroborated the pumping mechanism of den849 sity (due to a competition between mobility and 850 diffusion) and validated the assumption of density ${ }_{851}$ depletion in the flux tube connected to the probe. ${ }_{852}$ Nevertheless this assumption is not enough to make 853 appear the bump, the density decay in the flux tube ${ }_{854}$ must be stronger than the perpendicular expansion 855 of the flux tube with $V$, that is why the exponential 856 decay from our model is needed.

${ }_{857}$ We have finally compared different methods for ${ }_{858}$ extracting both $n_{e}$ and $T_{e}$ from bumped charac859 teristics, which are not very usual in the con860 text of probe measurements. We showed that the ${ }_{861}$ classical method of plasma potential determination ${ }_{862}$ (where $\mathrm{d} I / \mathrm{d} V$ is maximum) stays the most repro${ }_{863}$ ducible method to access this important parameter, 864 although previous studies argued that the plasma 865 potential coincides with the bump one. A lot of ${ }_{866}$ work is still needed to provide a complete theory ${ }_{867}$ that exploits bumped characteristics, especially to ${ }_{868}$ know the good collecting surfaces of the probe, and ${ }_{869}$ the good mobility and diffusion parameters to put 870 in the model.

\section{ACKNOWLEDGEMENTS}

This work has been carried out within the frame873 work of the French Federation for Magnetic Fusion ${ }_{874}$ Studies (FR-FCM) and of the Eurofusion consor875 tium, and has received funding from the Euratom 876 research and training programme 2014-2018 and 877 2019-2020 under grant agreement No 633053. The 878 views and opinions expressed herein do not neces879 sarily reflect those of the European Commission.

${ }^{1}$ M. A. Lieberman and A. J. Lichtenberg, Principles Of Plasma Discharges And Materials Processing, edited by Wiley (Wiley - Interscience, 2005).

${ }^{2} \mathrm{~F}$. Chen, Introduction to Plasma Physics and Controlled Fusion, edited by N. Y. Academic Press Inc. (Springer US, 1984).

${ }^{3}$ H. M. Mott-Smith and I. Langmuir, Physical Review 28, 727 (1926).

${ }^{4}$ J. Allen, R. Boyd, and P. Reynolds, Plasma Sources Science And Technology 18, 035012 (2009).

${ }^{5}$ J. Allen, Physica Scripta 45, 497 (1992)

${ }^{6}$ I. B. Bernstein and I. N. Rabinowitz, Physics Of Fluids 2, 119 (1959).

${ }^{7}$ I. D. Sudit and R. C. Woods, Journal of Applied Physics 76, 4488 (1994).

${ }^{8}$ J. Laframboise, UTIAS Report 10, 1 (1966)

${ }^{9}$ J. G. Laframboise and L. W. Parker, Physics Of Fluid 16, 629 (1973).

${ }^{10}$ J. G. Laframboise and J. Rubinstein, Physics Of Fluid 19, 1900 (1976).

${ }^{11}$ D. Bohm, E. Burhop, and H. Massey, "The use of probes for plasma exploration in strong magnetic fields," (MCGraw-Hill, New York, 1949) Chap. 2

${ }^{12}$ F. Chen, C. Etievant, and D. Mosher, Physics Of Fluids 11, $811(1968)$

${ }^{13}$ T. K. Popov, M. Dimitrova, P. Ivanova, J. Kovacic, T. Gyergyek, R. Dejarnac, J. Stocke, M. A. Pedrosa, D. Lopez-Bruna, and C. Hidalgo, Plasma Sources Science and Technology 25, 033001 (2016)

${ }^{14}$ J. Moritz, E. Faudot, S. Devaux, and S. Heuraux, Physics Of Plasmas 25, 013534 (2018)

${ }_{910}{ }^{15} \mathrm{~T}$. Dote and H. Amemiya, Journal Of The Physical Society Of Japan 19, 1915 (1964).

${ }^{16}$ I. Mihaila, M. Solomom, C. Costin, and G. Popa, Contribution Of Plasma Physics 53, 96 (2013).

${ }^{17}$ M. Usoltceva, E. Faudot, S. Devaux, S. Heuraux, J. Ledig, G. V. Zadvitskiy, R. Ochoukov, K. Crombe, and J.-M. Noterdaeme, Physics of Plasmas 25, 063518 (2018).

${ }_{917}^{18}$ M. Usoltceva, E. Faudot, J. Ledig, S. Devaux, S. Heuraux, G. V. Zadvitskiy, R. Ochoukov, J. Moritz, K. Crombe, and J.M. Noterdaeme, Review of Scientific Instruments 86, 10J124 (2018)

${ }^{19} \mathrm{~T}$. Dote and H. Amemiya, Journal Of The Physical Society Of Japan 22, 270 (1967)

${ }_{923}{ }^{20} \mathrm{~T}$. Dote and H. Amemiya, Japan Journal Applied Physics 8, 818 (1969)

${ }_{925}^{21}$ E. Faudot, Plasma Physics and Controlled Fusion 22, 083506 (2015).

${ }_{927}^{22} \mathrm{~K}$. Gunther and A. Carlson, Contribution On Plasma Physics 34, 484 (1994)

${ }_{929}{ }^{23} \mathrm{~V}$. Rozhansky, A. Ushakov, and S. Voskoboynikov, Nuclear Fusion 39, 613 (1999).

${ }_{931}{ }^{24} \mathrm{~A}$. Nedospasov and D. Uzdensky, Contributions To Plasma 932 Physics 34, 478 (1994).

$933{ }^{25} \mathrm{P}$. Verplancke, R. Chodura, J. Noterdaeme, and M. Weinlich, 934 Contributions To Plasma Physics 36, 145 (1996). 
${ }_{935}{ }^{26}$ E. Faudot, S. Devaux, J. Moritz, S. Heuraux, P. M. Cabrera, 947 neutral obtained from https://fr.lxcat.net (visited November 936 and F. Brochard, Review of Scientific Instruments 86, 063502 937 (2015) 948 11th 2018)

${ }_{938}{ }^{27}$ S.Devaux, E.Faudot, J.Moritz, and S.Heuraux, Nuclear Mate-

39 rials and Energy 12, 908 (2017)

${ }_{949}{ }^{31}$ E. Faudot, S. Heuraux, M. Kubic, J. Gunn, and L. Colas, $940{ }^{28}$ P. A. Chatterton, J. Rees, W. Wu, and K. Al-Assadi, Vacuum 950 Physics Of Plasmas 20, 043514 (2013).

$941 \quad$ 42, 489 (1991)

${ }_{951}{ }^{32} \mathrm{P}$. Chabert and N. Braithwaite, Physics Of Radio-Frequency

${ }^{29}$ F. Gerhard, Low Pressure Plasmas and Microstructuring Tech953 2011).

943 nology, edited by Springer (Springer-Verlag Berlin Heidel944 berg, 2009)

$945{ }^{30}$ L. Viehland, T. Skaist, C. Adhikari, and W. Siems, Contribu955 ical Review Letters, A. Valfells, L. K. Ang, and Y. Lau, Phys${ }_{956}{ }^{34} \mathrm{~F}$. Chen, Proceedings of the Physical Society. Section B 70 ,

946 tions To Plasma Physics 20, 1 (2017), scattering He ion/He 